\title{
NON-WEIGHT MODULES OVER THE AFFINE-VIRASORO ALGEBRA OF TYPE $A_{1}$
}

\author{
QIU-FAN CHEN AND JIAN-ZHI HAN
}

\begin{abstract}
In this paper, we study a class of non-weight modules over the affine-Virasoro algebra of type $A_{1}$, which are free modules of rank one when restricted to the Cartan subalgebra (modulo center). We give the classification of such modules. Moreover, the simplicity and the isomorphism classes of these modules are determined.
\end{abstract}

\section{INTRODUCTION}

The Virasoro algebra is an infinite dimensional Lie algebra over $\mathbb{C}$ with basis $\left\{d_{i}, C \mid i \in \mathbb{Z}\right\}$ and defining relations

$$
\left[d_{i}, d_{j}\right]=(j-i) d_{i+j}+\delta_{i+j, 0} \frac{i^{3}-i}{12} C, \quad\left[d_{i}, C\right]=0, \quad \forall i, j \in \mathbb{Z},
$$

which is the universal central extension of the so-called infinite dimensional Witt algebra of rank one. The Virasoro algebra occurs as the algebra of the conformal group in one dimension, or in the form of two commuting copies. Affine Lie algebras play an important role in string theory and two-dimensional conformal field theory. It is well known that the Virasoro algebra and the affine Lie algebras have been widely used in many physics areas and mathematical branches. Their close relationship strongly suggests that they should be considered simultaneously, that is, as one algebraic structure. Actually this has led to the definition of the so-called affine-Virasoro algebra, which is the semidirect product of the Virasoro algebra and an affine Kac-Moody Lie algebra with a common center. AffineVirasoro algebras are very meaningful in the sense that they are closely connected to the conformal field theory. For example, the even part of $N=3$ superconformal algebra [10] is just the affine-Virasoro algebra of type $A_{1}$. Highest weight representations and integrable

2010 Mathematics Subject Classification. 17B10, 17B35, 17B65, 17B68.

Key words and phrases. Affine-Virasoro algebra, non-weight module, free module.

This work is supported by National Natural Science Foundation of China (Grant Nos. 11801363, 11771279 and 11671247). 
representations of the affine-Virasoro algebras have been extensively studied (cf. [11], [15][18], [27]). The author in [2] presented the classification of all simple Harish-Chandra modules with nonzero central actions over the affine-Virasoro algebras. However, all simple uniform bounded modules over these algebras are not yet classified except the affine-Virasoro algebra of type $A_{1}[12$.

In recent years, many authors constructed various simple non-Harish-Chandra modules and simple non-weight modules (cf. [1], 3]-[9], [19]-[26]). In particular, J. Nilsson [23] constructed a class of $\mathfrak{s l}_{\mathfrak{n}+1}$-modules that are free of rank one when restricted to the Cartan subalgebra. This kind of non-weight modules, which many authors call $U(\mathfrak{h})$-free modules, have been extensively studied. In the paper [23] and a subsequent paper [24], J. Nilsson showed that a finite dimensional simple Lie algebra has nontrivial $U(\mathfrak{h})$-free modules if and only if it is of type $A$ or $C$. Furthermore, the $U(\mathfrak{h})$-free modules of rank one for the KacMoody Lie algebras were determined in [3]. And the idea was exploited and generalized to consider modules over infinite dimensional Lie algebras, such as the Witt algebras of arbitrary rank [26], Heisenberg-Virasoro algebra and $W(2,2)$ algebra [6], the algebras $\operatorname{Vir}(a, b)$ [14, the Lie algebras related to the Virasoro algebra [8, 9] and so on. The aim of this paper is to classify such modules for the the affine-Virasoro algebra of type $A_{1}$.

This paper is organized as follows. In Section 2, we construct a class of non-weight modules over the affine-Virasoro algebra of type $A_{1}$, and study the simplicity and isomorphic relations of these modules. Section 3 is devoted to classifying all modules whose restriction to the Cartan subalgebra (modulo center) are free of rank one over the affine-Virasoro algebra of type $A_{1}$.

Throughout the paper, we denote by $\mathbb{C}, \mathbb{Z}, \mathbb{C}^{*}, \mathbb{Z}_{+}$the sets of complex numbers, integers, nonzero complex numbers and nonnegative integers, respectively. All vector spaces are assumed to be over $\mathbb{C}$. For a Lie algebra $\mathfrak{g}$, we use $U(\mathfrak{g})$ to denote the universal enveloping algebra of $\mathfrak{g}$.

\section{Preliminaries}

In this section, we shall introduce some basic notations and establish some related results. 
Definition 2.1. Let $L$ be a finite-dimensional Lie algebra with a non-degenerated invariant symmetric bilinear form $(\cdot, \cdot)$. The affine-Virasoro algebra $L_{a v}$ is the vector space

$$
L_{a v}=L \otimes \mathbb{C}\left[t, t^{-1}\right] \oplus \mathbb{C} C \oplus \bigoplus_{i \in \mathbb{Z}} \mathbb{C} d_{i}
$$

with the Lie brackets:

$$
\begin{aligned}
& {\left[x \otimes t^{m}, y \otimes t^{n}\right]=[x, y] \otimes t^{m+n}+m(x, y) \delta_{m+n, 0} C,} \\
& {\left[d_{i}, d_{j}\right]=(j-i) d_{i+j}+\delta_{i+j, 0} \frac{i^{3}-i}{12} C,} \\
& {\left[d_{i}, x \otimes t^{m}\right]=m x \otimes t^{m+i}, \quad\left[C, L_{a v}\right]=0,}
\end{aligned}
$$

where $x, y \in L, m, n, i, j \in \mathbb{Z}$ (if $L$ has no such form, we set $(x, y)=0$ for all $x, y \in L$ ).

We see that if $L=\mathbb{C} e$ is one-dimensional, then $L_{a v}$ is just the twisted Heisenberg-Virasoro algebra (one center element). In the following we only consider specially $L$ as the simple Lie algebra $\mathfrak{s l}_{2}=\operatorname{span}_{\mathbb{C}}\{e, f, h\}$.

Definition 2.2. Take $L$ to be the Lie algebra $\mathfrak{s l}_{2}$ in Definition 2.1. Then the resulting Lie algebra $\mathfrak{L}$ (here the bilinear form $(\cdot, \cdot)$ is normalized by $(e, f)=1$ ) with $\mathbb{C}$-basis $\left\{e_{i}, f_{i}, h_{i}, d_{i}, C \mid i \in \mathbb{Z}\right\}$ subject to the following Lie brackets:

$$
\begin{aligned}
& {\left[e_{i}, f_{j}\right]=h_{i+j}+i \delta_{i+j, 0} C,} \\
& {\left[h_{i}, e_{j}\right]=2 e_{i+j}, \quad\left[h_{i}, f_{j}\right]=-2 f_{i+j},} \\
& {\left[d_{i}, d_{j}\right]=(j-i) d_{i+j}+\delta_{i+j, 0} \frac{i^{3}-i}{12} C,} \\
& {\left[d_{i}, h_{j}\right]=j h_{i+j}, \quad\left[h_{i}, h_{j}\right]=-2 i \delta_{i+j, 0} C,} \\
& {\left[d_{i}, e_{j}\right]=j e_{i+j}, \quad\left[d_{i}, f_{j}\right]=j f_{i+j},} \\
& {\left[e_{i}, e_{j}\right]=\left[f_{i}, f_{j}\right]=[C, \mathfrak{L}]=0,}
\end{aligned}
$$

is called the affine-Virasoro algebra of type $A_{1}$.

Definition 2.3. Let $\mathbb{C}[s, t]$ be the polynomial algebra in variables $s$ and $t$ with coefficients in $\mathbb{C}$. For $\lambda, \alpha \in \mathbb{C}^{*}, \beta, \gamma \in \mathbb{C}, i \in \mathbb{Z}$ and $g(s, t) \in \mathbb{C}[s, t]$, define the action of $\mathfrak{L}$ on $\mathbb{C}[s, t]$ as follows:

$$
\begin{aligned}
\Omega(\lambda, \alpha, \beta, \gamma): \quad e_{i} \cdot g(s, t) & =\lambda^{i} \alpha g(s-i, t-2) \\
f_{i} \cdot g(s, t) & =-\frac{\lambda^{i}}{\alpha}\left(\frac{t}{2}-\beta\right)\left(\frac{t}{2}+\beta+1\right) g(s-i, t+2)
\end{aligned}
$$




$$
\begin{aligned}
h_{i} \cdot g(s, t) & =\lambda^{i} \operatorname{tg}(s-i, t), \quad d_{i} \cdot g(s, t)=\lambda^{i}(s+i \gamma) g(s-i, t), \\
C \cdot g(s, t) & =0 ; \\
\Delta(\lambda, \alpha, \beta, \gamma): \quad e_{i} \cdot g(s, t) & =-\frac{\lambda^{i}}{\alpha}\left(\frac{t}{2}+\beta\right)\left(\frac{t}{2}-\beta-1\right) g(s-i, t-2), \\
f_{i} \cdot g(s, t) & =\lambda^{i} \alpha g(s-i, t+2), \quad h_{i} \cdot g(s, t)=\lambda^{i} t g(s-i, t), \\
d_{i} \cdot g(s, t) & =\lambda^{i}(s+i \gamma) g(s-i, t), \\
C \cdot g(s, t) & =0 ; \\
\Theta(\lambda, \alpha, \beta, \gamma): \quad e_{i} \cdot g(s, t) & =\lambda^{i} \alpha\left(\frac{t}{2}+\beta\right) g(s-i, t-2), \\
f_{i} \cdot g(s, t) & =-\frac{\lambda^{i}}{\alpha}\left(\frac{t}{2}-\beta\right) g(s-i, t+2), \\
h_{i} \cdot g(s, t) & =\lambda^{i} t g(s-i, t), \quad d_{i} \cdot g(s, t)=\lambda^{i}(s+i \gamma) g(s-i, t), \\
C \cdot g(s, t) & =0 .
\end{aligned}
$$

Remark 2.4. (1) Whenever we consider the action of $\mathfrak{L}$ on $\mathbb{C}[s, t]$, we always mean one of these above.

(2) Denote by $\mathfrak{H}_{h}$ the vector space spanned by the set $\left\{h_{i}, d_{i}, C \mid i \in \mathbb{Z}\right\}$. An important fact needs to be pointed here is: though $\mathfrak{H}_{h}$ is an quotient algebra of the Heisenberg-Virasoro algebra $\operatorname{Vir}(0,0)$, they have the same submodule structure on $\mathbb{C}[s, t]$ (cf. [6] or [14]).

Proposition 2.5. $\Omega(\lambda, \alpha, \beta, \gamma), \Delta(\lambda, \alpha, \beta, \gamma)$ and $\Theta(\lambda, \alpha, \beta, \gamma)$ are $\mathfrak{L}$-modules under the actions given in Definition [2.3. Moreover, $\Omega(\lambda, \alpha, \beta, \gamma)$ and $\Delta(\lambda, \alpha, \beta, \gamma)$ are simple for all $\lambda, \alpha \in \mathbb{C}^{*}$ and $\beta, \gamma \in \mathbb{C} ; \Theta(\lambda, \alpha, \beta, \gamma)$ is simple if and only if $2 \beta \notin \mathbb{Z}_{+}$.

Proof. For the first statement, we only tackle the case $\Omega(\lambda, \alpha, \beta, \gamma)$, since the other two cases can be treated similarly. In view of the $\mathfrak{H}_{h}$-action, we know the following relations

$$
\begin{aligned}
d_{i} \circ d_{j}-d_{j} \circ d_{i} & =\left[d_{i}, d_{j}\right], \\
d_{i} \circ h_{j}-h_{j} \circ d_{i} & =\left[d_{i}, h_{j}\right], \\
h_{i} \circ h_{j}-h_{j} \circ h_{i} & =\left[h_{i}, h_{j}\right]
\end{aligned}
$$

hold on $\Omega(\lambda, \alpha, \beta, \gamma)$ by [14, Proposition 2.2]. Note according to the above definition for any $g(s, t) \in \mathbb{C}[s, t]$ that

$$
e_{i} \cdot f_{j} \cdot g(s, t)-f_{j} \cdot e_{i} \cdot g(s, t)
$$




$$
\begin{aligned}
& =e_{i} \cdot\left(-\frac{\lambda^{j}}{\alpha}\left(\frac{t}{2}-\beta\right)\left(\frac{t}{2}+\beta+1\right) g(s-j, t+2)\right) \\
& -f_{j} \cdot\left(\lambda^{i} \alpha g(s-i, t-2)\right) \\
& =-\lambda^{i+j}\left(\frac{t}{2}-\beta-1\right)\left(\frac{t}{2}+\beta\right) g(s-i-j, t) \\
& +\lambda^{i+j}\left(\frac{t}{2}-\beta\right)\left(\frac{t}{2}+\beta+1\right) g(s-i-j, t) \\
& =\lambda^{i+j} \operatorname{tg}(s-i-j, t) \\
& =\left(h_{i+j}+i \delta_{i+j, 0} C\right) \cdot g(s, t)=\left[e_{i}, f_{j}\right] \cdot g(s, t), \\
& h_{i} \cdot e_{j} \cdot g(s, t)-e_{j} \cdot h_{i} \cdot g(s, t) \\
& =h_{i} \cdot\left(\lambda^{j} \alpha g(s-j, t-2)\right)-e_{j} \cdot\left(\lambda^{i} \operatorname{tg}(s-i, t)\right) \\
& =\lambda^{i+j} \alpha t g(s-i-j, t-2)-\lambda^{i+j} \alpha(t-2) g(s-i-j, t-2) \\
& =2 \lambda^{i+j} \alpha g(s-i-j, t-2) \\
& =2 e_{i+j} \cdot g(s, t)=\left[h_{i}, e_{j}\right] \cdot g(s, t) \text {, } \\
& h_{i} \cdot f_{j} \cdot g(s, t)-f_{j} \cdot h_{i} \cdot g(s, t) \\
& =h_{i} \cdot\left(-\frac{\lambda^{j}}{\alpha}\left(\frac{t}{2}-\beta\right)\left(\frac{t}{2}+\beta+1\right) g(s-j, t+2)\right) \\
& -f_{j} \cdot\left(\lambda^{i} \operatorname{tg}(s-i, t)\right) \\
& =-\frac{\lambda^{i+j}}{\alpha} t\left(\frac{t}{2}-\beta\right)\left(\frac{t}{2}+\beta+1\right) g(s-i-j, t+2) \\
& +\frac{\lambda^{i+j}}{\alpha}\left(\frac{t}{2}-\beta\right)\left(\frac{t}{2}+\beta+1\right)(t+2) g(s-i-j, t+2) \\
& =\frac{2 \lambda^{i+j}}{\alpha}\left(\frac{t}{2}-\beta\right)\left(\frac{t}{2}+\beta+1\right) g(s-i-j, t+2) \\
& =-2 f_{i+j} \cdot g(s, t)=\left[h_{i}, f_{j}\right] \cdot g(s, t) \text {, } \\
& d_{i} \cdot e_{j} \cdot g(s, t)-e_{j} \cdot d_{i} \cdot g(s, t) \\
& =d_{i} \cdot\left(\lambda^{j} \alpha g(s-j, t-2)\right)-e_{j} \cdot\left(\lambda^{i}(s+i \gamma) g(s-i, t)\right) \\
& =\lambda^{i+j} \alpha(s+i \gamma) g(s-i-j, t-2)-\lambda^{i+j} \alpha(s+i \gamma-j) g(s-i-j, t-2) \\
& =j \lambda^{i+j} \alpha g(s-i-j, t-2) \\
& =j e_{i+j} \cdot g(s, t)=\left[d_{i}, e_{j}\right] \cdot g(s, t)
\end{aligned}
$$


and

$$
\begin{aligned}
& d_{i} \cdot f_{j} \cdot g(s, t)-f_{j} \cdot d_{i} \cdot g(s, t) \\
= & d_{i} \cdot\left(-\frac{\lambda^{j}}{\alpha}\left(\frac{t}{2}-\beta\right)\left(\frac{t}{2}+\beta+1\right) g(s-j, t+2)\right) \\
& -f_{j} \cdot\left(\lambda^{i}(s+i \gamma) g(s-i, t)\right) \\
= & -\frac{\lambda^{i+j}}{\alpha}(s+i \gamma)\left(\frac{t}{2}-\beta\right)\left(\frac{t}{2}+\beta+1\right) g(s-i-j, t+2) \\
& +\frac{\lambda^{i+j}}{\alpha}(s+i \gamma-j)\left(\frac{t}{2}-\beta\right)\left(\frac{t}{2}+\beta+1\right) g(s-i-j, t+2) \\
= & -\frac{j \lambda^{i+j}}{\alpha}\left(\frac{t}{2}-\beta\right)\left(\frac{t}{2}+\beta+1\right) g(s-i-j, t+2) \\
= & j f_{i+j} \cdot g(s, t)=\left[d_{i}, f_{j}\right] \cdot g(s, t) .
\end{aligned}
$$

And the last three relations

$$
\begin{array}{ll} 
& e_{i} \circ e_{j}-e_{j} \circ e_{i}=\left[e_{i}, e_{j}\right], \\
& f_{i} \circ f_{j}-f_{j} \circ f_{i}=\left[f_{i}, f_{j}\right], \\
\text { and } \quad & C \circ x-x \circ C=0, \forall x \in \mathcal{L}
\end{array}
$$

can be checked easily, proving the first statement.

Note that $d_{0}$ and $h_{0}$ are in fact the left multiplication operators $s$ and $t$ on $\mathbb{C}[s, t]$. In particular, 1 is a generator of the $\mathfrak{L}$-module $\mathbb{C}[s, t]$. So the simplicity of these modules is equivalent to determining whether every nonzero $\mathfrak{L}$-submodule $\mathbb{C}[s, t]$ contains 1 . Let $W$ be a nonzero submodule of $\Omega(\lambda, \alpha, \beta, \gamma), \Delta(\lambda, \alpha, \beta, \gamma)$ or $\Theta(\lambda, \alpha, \beta, \gamma)$. Then regarding as an $\mathfrak{H}_{h}$-submodule, either $W=\mathbb{C}[s, t] g(t)$ or $W=\mathbb{C}[s, t] s g(t)+\mathbb{C}[s, t] \operatorname{tg}(t)$ for some nonzero polynomial $g(t) \in \mathbb{C}[t]$ by [13, Theorem 2.3] and Remark 2.4(2).

Claim 1. $1 \in W$ if $W \subseteq \Omega(\lambda, \alpha, \beta, \gamma)(\operatorname{resp} . \Delta(\lambda, \alpha, \beta, \gamma))$

Consider $W \subseteq \Omega(\lambda, \alpha, \beta, \gamma)$. If $W=\mathbb{C}[s, t] g(t)$, then from the definition of the module structure one can inductively show that

$$
g(t-2 k)=\alpha^{-k} e_{0}^{k} \cdot g(t) \in W .
$$

Note that we can make $g(t)$ and $g(t-2 k)$ coprime by choosing $k$ large enough.

If $W=\mathbb{C}[s, t] s g(t)+\mathbb{C}[s, t] \operatorname{tg}(t)$, then using induction on $k$ one has

$$
\left(\lambda e_{0}\right)^{k} \cdot s g(t)=(\lambda \alpha)^{k} s g(t-2 k),
$$




$$
e_{1}^{k} \cdot s g(t)=(\lambda \alpha)^{k}(s-k) g(t-2 k),
$$

and the subtraction of these two gives

$$
g(t-2 k)=\frac{1}{k(\lambda \alpha)^{k}}\left(\left(\lambda e_{0}\right)^{k} \cdot s g(t)-e_{1}^{k} \cdot s g(t)\right) \in W .
$$

It is easy to see that $t g(t)$ and $g(t-2 k)$ coprime by choosing $k$ large enough. That is, we have coprime elements in $W$ and therefore $1 \in W$ in both cases. Using the similar argument as for $\Omega(\lambda, \alpha, \beta, \gamma)$ but respectively replacing $e_{0}, e_{1}$ with $f_{0}, f_{1}$ we see that $1 \in W$ if $W \subseteq \Delta(\lambda, \alpha, \beta, \gamma)$.

Now assume that $W \subseteq \Theta(\lambda, \alpha, \beta, \gamma)$.

Claim 2. $1 \in W$ if $2 \beta \notin \mathbb{Z}_{+}$and there exists a nonzero (simple) $\mathcal{L}$-submodule $V$ of $\Theta(\lambda, \alpha, \beta, \gamma)$ such that $1 \notin V$ if $2 \beta \in \mathbb{Z}_{+}$.

If $W=\mathbb{C}[s, t] g(t)$, then using induction on $k$ one immediately has

$$
\begin{gathered}
e_{0}^{k} \cdot g(t)=\alpha^{k} \prod_{n=0}^{k-1}\left(\frac{t}{2}+\beta-n\right) g(t-2 k), \\
f_{0}^{k} \cdot g(t)=\left(-\frac{1}{\alpha}\right)^{k} \prod_{n=0}^{k-1}\left(\frac{t}{2}-\beta+n\right) g(t+2 k) .
\end{gathered}
$$

And for the other case $W=\mathbb{C}[s, t] s g(t)+\mathbb{C}[s, t] \operatorname{tg}(t)$, one has

$$
\begin{gathered}
e_{0}^{k} \cdot \operatorname{tg}(t)=\alpha^{k} \prod_{n=0}^{k-1}\left(\frac{t}{2}+\beta-n\right)(t-2 k) g(t-2 k), \\
f_{0}^{k} \cdot \operatorname{tg}(t)=\left(-\frac{1}{\alpha}\right)^{k} \prod_{n=0}^{k-1}\left(\frac{t}{2}-\beta+n\right)(t+2 k) g(t+2 k) .
\end{gathered}
$$

Note that if $2 \beta \notin \mathbb{Z}_{+}$, then $e_{0}^{k} \cdot g(t)$ and $f_{0}^{k} \cdot g(t)$ (resp. $e_{0}^{k} \cdot t g(t)$ and $f_{0}^{k} \cdot t g(t)$ ) for large enough $k$ are coprime elements in $W$. Thus, $1 \in W$.

Assume that $2 \beta \in \mathbb{Z}_{+}$. Consider the vector space

$$
V:=\mathbb{C}[s, t] \prod_{n=0}^{2 \beta}\left(\frac{t}{2}+\beta-n\right) .
$$

Clearly,

$$
\mathfrak{H}_{h} \cdot V \subset V .
$$

For any $0 \neq g(s, t) \prod_{n=0}^{2 \beta}\left(\frac{t}{2}+\beta-n\right) \in V$ and $i \in \mathbb{Z}$, we have

$$
e_{i} \cdot g(s, t) \prod_{n=0}^{2 \beta}\left(\frac{t}{2}+\beta-n\right)=\lambda^{i} \alpha\left(\frac{t}{2}+\beta\right) g(s-i, t-2) \prod_{n=0}^{2 \beta}\left(\frac{t}{2}+\beta-n-1\right)
$$




$$
=\lambda^{i} \alpha\left(\frac{t}{2}-\beta-1\right) g(s-i, t-2) \prod_{n=0}^{2 \beta}\left(\frac{t}{2}+\beta-n\right)
$$

and

$$
\begin{aligned}
f_{i} \cdot g(s, t) \prod_{n=0}^{2 \beta}\left(\frac{t}{2}+\beta-n\right) & =-\frac{\lambda^{i}}{\alpha}\left(\frac{t}{2}-\beta\right) g(s-i, t+2) \prod_{n=0}^{2 \beta}\left(\frac{t}{2}+\beta-n+1\right) \\
& =-\frac{\lambda^{i}}{\alpha}\left(\frac{t}{2}+\beta+1\right) g(s-i, t+2) \prod_{n=0}^{2 \beta}\left(\frac{t}{2}+\beta-n\right),
\end{aligned}
$$

These along with (2.1) show that $V$ is a proper submodule. Furthermore, (2.2) and (2.3) entail us to establish an $\mathfrak{L}$-module isomorphism

$$
\tau: \Theta(\lambda, \alpha,-\beta-1, \gamma) \rightarrow V \quad g(s, t) \mapsto g(s, t) \prod_{n=0}^{2 \beta}\left(\frac{t}{2}+\beta-n\right) .
$$

Then $V \cong \Theta(\lambda, \alpha,-\beta-1, \gamma)$ is simple, since $2(-\beta-1) \notin \mathbb{Z}_{+}$. This completes the proof.

The following proposition gives a characterization of isomorphisms between the $\mathfrak{L}$-modules constructed above.

Proposition 2.6. Let $\lambda, \lambda^{\prime}, \alpha, \alpha^{\prime} \in \mathbb{C}^{*}, \beta, \beta^{\prime}, \gamma, \gamma^{\prime} \in \mathbb{C}$. Then

$$
\begin{aligned}
& \Omega(\lambda, \alpha, \beta, \gamma) \cong \Omega\left(\lambda^{\prime}, \alpha^{\prime}, \beta^{\prime}, \gamma^{\prime}\right) \Longleftrightarrow(\lambda, \alpha, \beta, \gamma)=\left(\lambda^{\prime}, \alpha^{\prime}, \beta^{\prime}, \gamma^{\prime}\right) \\
& \text { or } \quad(\lambda, \alpha, \beta, \gamma)=\left(\lambda^{\prime}, \alpha^{\prime},-\beta^{\prime}-1, \gamma^{\prime}\right) ; \\
& \Delta(\lambda, \alpha, \beta, \gamma) \cong \Delta\left(\lambda^{\prime}, \alpha^{\prime}, \beta^{\prime}, \gamma^{\prime}\right) \Longleftrightarrow(\lambda, \alpha, \beta, \gamma)=\left(\lambda^{\prime}, \alpha^{\prime}, \beta^{\prime}, \gamma^{\prime}\right) \\
& \text { or }(\lambda, \alpha, \beta, \gamma)=\left(\lambda^{\prime}, \alpha^{\prime},-\beta^{\prime}-1, \gamma^{\prime}\right) ; \\
& \Theta(\lambda, \alpha, \beta, \gamma) \cong \Theta\left(\lambda^{\prime}, \alpha^{\prime}, \beta^{\prime} \gamma^{\prime}\right) \Longleftrightarrow(\lambda, \alpha, \beta, \gamma)=\left(\lambda^{\prime}, \alpha^{\prime}, \beta^{\prime}, \gamma^{\prime}\right) .
\end{aligned}
$$

Proof. We only prove (2.4), a similar argument can be applied to (2.5) and (2.6). For this, it suffices to show the " $\Longrightarrow$ " part. Let $\varphi: \Omega(\lambda, \alpha, \beta, \gamma) \rightarrow \Omega\left(\lambda^{\prime}, \alpha^{\prime}, \beta^{\prime}, \gamma^{\prime}\right)$ be an isomorphism of $\mathfrak{L}$-modules. Viewing $\Omega(\lambda, \alpha, \beta, \gamma)$ and $\Omega\left(\lambda^{\prime}, \alpha^{\prime}, \beta^{\prime}, \gamma^{\prime}\right)$ as $\mathfrak{H}_{h}$-modules, we get $(\lambda, \gamma)=\left(\lambda^{\prime}, \gamma^{\prime}\right)$ by [14, Proposition 2.3(ii)]. Now for any $g(t) \in \mathbb{C}[t]$, we have

$$
\begin{gathered}
\varphi(g(t))=\varphi\left(g\left(h_{0}\right) \cdot 1\right)=g\left(h_{0}\right) \cdot \varphi(1)=g(t) \varphi(1), \\
\varphi(\alpha)=\varphi\left(e_{0} \cdot 1\right)=e_{0} \cdot \varphi(1)=\alpha^{\prime} \varphi(1)
\end{gathered}
$$

and $\varphi\left(-\frac{1}{\alpha}\left(\frac{t}{2}-\beta\right)\left(\frac{t}{2}+\beta+1\right)\right)=\varphi\left(f_{0} \cdot 1\right)=f_{0} \cdot \varphi(1)=-\frac{1}{\alpha^{\prime}}\left(\frac{t}{2}-\beta^{\prime}\right)\left(\frac{t}{2}+\beta^{\prime}+1\right) \varphi(1)$. 
It is easy to see from the first two formulae above that $\alpha=\alpha^{\prime}$, which together with the first and third formulae gives rise to $\beta=\beta^{\prime}$ or $\beta=-\beta^{\prime}-1$. This completes the proof.

\section{MAIN RESUlT}

It is clear that the Cartan subalgebra (modulo center) of $\mathfrak{L}$ is spanned by $h_{0}$ and $d_{0}$. The main result of the present paper is to classify all modules over $\mathfrak{L}$ whose restrictions to $U\left(\mathbb{C} d_{0} \oplus \mathbb{C} h_{0}\right)$ are free of rank 1 . Before presenting the main result, we first give a lemma, which can be easily shown by induction on $m$.

Lemma 3.1. For any $i \in \mathbb{Z}$ and $0 \leq m \in \mathbb{Z}$, we have

$$
\begin{aligned}
e_{i} d_{0}^{m}=\left(d_{0}-i\right)^{m} e_{i}, & f_{i} d_{0}^{m}=\left(d_{0}-i\right)^{m} f_{i}, \\
e_{i} h_{0}^{m}=\left(h_{0}-2\right)^{m} e_{i}, & f_{i} h_{0}^{m}=\left(h_{0}+2\right)^{m} f_{i} .
\end{aligned}
$$

Theorem 3.2. Any $U(\mathfrak{L})$-module $M$ such that its restriction to $U\left(\mathbb{C} d_{0} \oplus \mathbb{C} h_{0}\right)$ is free of rank 1 is isomorphic to one of the modules

$$
\Omega(\lambda, \alpha, \beta, \gamma), \Delta(\lambda, \alpha, \beta, \gamma), \Theta(\lambda, \alpha, \beta, \gamma)
$$

for some $\lambda, \alpha \in \mathbb{C}^{*}$ and $\beta, \gamma \in \mathbb{C}$.

Proof. Let $M$ be an $\mathfrak{L}$-module which is a free $U\left(\mathbb{C} d_{0} \oplus \mathbb{C} h_{0}\right)$-module of rank 1 . Then $M=$ $U\left(\mathbb{C} d_{0} \oplus \mathbb{C} h_{0}\right)$. It follows from viewing as an $\mathfrak{H}_{h}$-module that $M \cong \Phi(\lambda, 0, \mathbf{p})$ by [14, Theorem $3.1]$ :

$$
\begin{aligned}
& d_{i} \cdot g\left(d_{0}, h_{0}\right)=\lambda^{i}\left(d_{0}+p_{i}\left(h_{0}\right)\right) g\left(d_{0}-i, h_{0}\right), \\
& h_{i} \cdot g\left(d_{0}, h_{0}\right)=\lambda^{i} h_{0} g\left(d_{0}-i, h_{0}\right), \quad C \cdot g\left(d_{0}, h_{0}\right)=0,
\end{aligned}
$$

where $g\left(d_{0}, h_{0}\right) \in U\left(\mathbb{C} d_{0} \oplus \mathbb{C} h_{0}\right), \lambda \in \mathbb{C}^{*}, i \in \mathbb{Z}$ and

$$
\mathbf{p}=\left(p_{i}\left(h_{0}\right)\right)_{i \in \mathbb{Z}} \in\left\{\left(p_{i}\left(h_{0}\right)\right)_{i \in \mathbb{Z}} \mid p_{i}\left(h_{0}\right)=\sum_{l=0}^{\infty} p^{(l)} i h_{0}^{l} \in \mathbb{C}\left[h_{0}\right], p^{(l)} \in \mathbb{C}\right\} .
$$

For any $i \in \mathbb{Z}$, assume that $E_{i}\left(d_{0}, h_{0}\right)=e_{i} \cdot 1$ and $F_{i}\left(d_{0}, h_{0}\right)=f_{i} \cdot 1$ for some $E_{i}\left(d_{0}, h_{0}\right)$, $F_{i}\left(d_{0}, h_{0}\right) \in U\left(\mathbb{C} d_{0} \oplus \mathbb{C} h_{0}\right)$. Take any

$$
g\left(d_{0}, h_{0}\right)=\sum_{j, k \in \mathbb{Z}_{+}} g_{j, k} d_{0}^{j} h_{0}^{k} \in U\left(\mathbb{C} d_{0} \oplus \mathbb{C} h_{0}\right), \quad \text { where } g_{j, k} \in \mathbb{C} .
$$


Then by Lemma 3.1,

$$
\begin{aligned}
e_{i} \cdot g\left(d_{0}, h_{0}\right) & =e_{i} \cdot \sum_{j, k \in \mathbb{Z}_{+}} g_{j, k} d_{0}^{j} h_{0}^{k}=\sum_{j, k \in \mathbb{Z}_{+}} g_{j, k}\left(d_{0}-i\right)^{j} e_{i} \cdot h_{0}^{k} \\
& =\sum_{j, k \in \mathbb{Z}_{+}} g_{j, k}\left(d_{0}-i\right)^{j}\left(h_{0}-2\right)^{k} E_{i}\left(d_{0}, h_{0}\right)
\end{aligned}
$$

and

$$
\begin{aligned}
f_{i} \cdot g\left(d_{0}, h_{0}\right) & =f_{i} \cdot \sum_{j, k \in \mathbb{Z}_{+}} g_{j, k} d_{0}^{j} h_{0}^{k}=\sum_{j, k \in \mathbb{Z}_{+}} g_{j, k}\left(d_{0}-i\right)^{j} f_{i} \cdot h_{0}^{k} \\
& =\sum_{j, k \in \mathbb{Z}_{+}} g_{j, k}\left(d_{0}-i\right)^{j}\left(h_{0}+2\right)^{k} F_{i}\left(d_{0}, h_{0}\right) .
\end{aligned}
$$

Thus, the actions of $e_{i}$ and $f_{i}$ on $M$ are completely determined by $E_{i}\left(d_{0}, h_{0}\right)$ and $F_{i}\left(d_{0}, h_{0}\right)$, respectively. For this reason, in what follows we only need to determine $E_{i}\left(d_{0}, h_{0}\right)$ and $F_{i}\left(d_{0}, h_{0}\right)$ for all $i \in \mathbb{Z}$.

Using (3.1) and (3.2), we present some formulae here, which will be used to do calculations in the following. The equations

$$
\left[e_{0}, f_{0}\right] \cdot 1=h_{0} \cdot 1,\left[e_{0}, e_{1}\right] \cdot 1=0,\left[f_{0}, f_{1}\right] \cdot 1=0
$$

are respectively equivalent to

$$
\begin{gathered}
E_{0}\left(d_{0}, h_{0}\right) F_{0}\left(d_{0}, h_{0}-2\right)-E_{0}\left(d_{0}, h_{0}+2\right) F_{0}\left(d_{0}, h_{0}\right)=h_{0}, \\
E_{0}\left(d_{0}, h_{0}\right) E_{1}\left(d_{0}, h_{0}-2\right)=E_{0}\left(d_{0}-1, h_{0}-2\right) E_{1}\left(d_{0}, h_{0}\right), \\
F_{0}\left(d_{0}, h_{0}\right) F_{1}\left(d_{0}, h_{0}+2\right)=F_{0}\left(d_{0}-1, h_{0}+2\right) F_{1}\left(d_{0}, h_{0}\right) .
\end{gathered}
$$

Note that $E_{0}\left(d_{0}, h_{0}\right) F_{0}\left(d_{0}, h_{0}\right) \neq 0$. Since otherwise $E_{0}\left(d_{0}, h_{0}\right) F_{0}\left(d_{0}, h_{0}\right)=0$ and (3.3) would give $h_{0}=0$, which is absurd. So we may assume

$$
E_{0}\left(d_{0}, h_{0}\right)=\sum_{i=0}^{m} a_{i}\left(d_{0}\right) h_{0}^{i} \quad \text { and } \quad F_{0}\left(d_{0}, h_{0}\right)=\sum_{i=0}^{n} b_{i}\left(d_{0}\right) h_{0}^{i}
$$

for some $a_{i}\left(d_{0}\right), b_{i}\left(d_{0}\right) \in \mathbb{C}\left[d_{0}\right]$ and $a_{m}\left(d_{0}\right) b_{n}\left(d_{0}\right) \neq 0$. Inserting these expressions into (3.3. yields

$$
\sum_{i=0}^{m} a_{i}\left(d_{0}\right) h_{0}^{i} \sum_{i=0}^{n} b_{i}\left(d_{0}\right)\left(h_{0}-2\right)^{i}-\sum_{i=0}^{m} a_{i}\left(d_{0}\right)\left(h_{0}+2\right)^{i} \sum_{i=0}^{n} b_{i}\left(d_{0}\right) h_{0}^{i}=h_{0}
$$


comparing highest degree terms, with respect to $h_{0}$, of both sides of which gives

$$
m+n=2 \quad \text { and } \quad a_{m}\left(d_{0}\right) b_{n}\left(d_{0}\right)=-\frac{1}{4} .
$$

From now on the discussion are divided into the following three cases.

Case 1. $(m, n)=(0,2)$.

In this case, we have

$$
E_{0}\left(d_{0}, h_{0}\right)=\alpha \quad \text { and } \quad F_{0}\left(d_{0}, h_{0}\right)=-\frac{1}{4 \alpha} h_{0}^{2}+u_{1}\left(d_{0}\right) h_{0}+v_{1}\left(d_{0}\right)
$$

for some $\alpha \in \mathbb{C}^{*}$ and $u_{1}\left(d_{0}\right), v_{1}\left(d_{0}\right) \in \mathbb{C}\left[d_{0}\right]$. Inserting the two expressions into (3.3) one has $u_{1}\left(d_{0}\right)=-\frac{1}{2 \alpha}$. Namely,

$$
F_{0}\left(d_{0}, h_{0}\right)=-\frac{1}{4 \alpha} h_{0}^{2}-\frac{1}{2 \alpha} h_{0}+v_{1}\left(d_{0}\right) .
$$

It follows from this and $\left[h_{1}, f_{0}\right] \cdot 1=-2 f_{1} \cdot 1$ that

$$
F_{1}\left(d_{0}, h_{0}\right)=\lambda\left(-\frac{1}{4 \alpha} h_{0}^{2}-\frac{1}{2 \alpha} h_{0}+\frac{1}{2} h_{0} v_{1}\left(d_{0}\right)-\frac{1}{2} h_{0} v_{1}\left(d_{0}-1\right)+v_{1}\left(d_{0}\right)\right) .
$$

Inserting (3.6), (3.7) into (3.5) and equating the terms do not depend on $h_{0}$ of both sides, we obtain

$$
v_{1}\left(d_{0}\right)^{2}=v_{1}\left(d_{0}\right) v_{1}\left(d_{0}-1\right)
$$

which forces $v_{1}\left(d_{0}\right) \in \mathbb{C}$. So

$$
F_{0}\left(d_{0}, h_{0}\right)=-\frac{1}{\alpha}\left(\frac{h_{0}}{2}-\beta\right)\left(\frac{h_{0}}{2}+\beta+1\right) \quad \text { for some } \beta \in \mathbb{C} .
$$

Then

$$
\begin{aligned}
& E_{i}\left(d_{0}, h_{0}\right)=e_{i} \cdot 1=\frac{1}{2}\left[h_{i}, e_{0}\right] \cdot 1=\frac{1}{2}\left(\alpha h_{i} \cdot 1-\lambda^{i} e_{0} \cdot h_{0} \cdot 1\right)=\frac{1}{2}\left(\alpha \lambda^{i} h_{0}-\alpha \lambda^{i}\left(h_{0}-2\right)\right)=\alpha \lambda^{i}, \\
& F_{i}\left(d_{0}, h_{0}\right)= f_{i} \cdot 1=\frac{1}{2}\left[f_{0}, h_{i}\right] \cdot 1=\frac{1}{2}\left(\lambda^{i} f_{0} \cdot h_{0} \cdot 1-h_{i} \cdot F_{0}\left(d_{0}, h_{0}\right)\right) \\
&=\frac{\lambda^{i}}{2}\left(\left(h_{0}+2\right) F_{0}\left(d_{0}, h_{0}\right)-h_{0} F_{0}\left(d_{0}-i, h_{0}\right)\right) \\
&=-\frac{\lambda^{i}}{\alpha}\left(\frac{h_{0}}{2}-\beta\right)\left(\frac{h_{0}}{2}+\beta+1\right), \quad \forall i \in \mathbb{Z} .
\end{aligned}
$$

And from $\left[d_{i}, e_{0}\right] \cdot 1=0$ we can deduce that $p_{i}\left(h_{0}\right) \in \mathbb{C}$, i.e., $p_{i}\left(h_{0}\right)=i \gamma$ for some $\gamma \in \mathbb{C}$ and all $i \in \mathbb{Z}$. Thus, $M \cong \Omega(\lambda, \alpha, \beta, \gamma)$. 
Case 2. $(m, n)=(2,0)$.

Interchanging $E_{0}\left(d_{0}, h_{0}\right)$ and $F_{0}\left(d_{0}, h_{0}\right)$ and then imitating the proof of Case 1 , we will see that $M \cong \Delta(\lambda, \alpha, \beta, \gamma)$.

Case 3. $(m, n)=(1,1)$.

Now we can assume that

$$
E_{0}\left(d_{0}, h_{0}\right)=\frac{\alpha}{2} h_{0}+u_{2}\left(d_{0}\right)
$$

and

$$
F_{0}\left(d_{0}, h_{0}\right)=-\frac{1}{2 \alpha} h_{0}+v_{2}\left(d_{0}\right)
$$

for some $\alpha \in \mathbb{C}^{*}$ and $u_{2}\left(d_{0}\right), v_{2}\left(d_{0}\right) \in \mathbb{C}\left[d_{0}\right]$. Inserting the two expressions into (3.3) forces

$$
u_{2}\left(d_{0}\right)=\alpha^{2} v_{2}\left(d_{0}\right)
$$

It follows from (3.8) and $\left[h_{1}, e_{0}\right] \cdot 1=2 e_{1} \cdot 1$ that

$$
E_{1}\left(d_{0}, h_{0}\right)=\lambda\left(\frac{\alpha}{2} h_{0}+\frac{1}{2} h_{0} u_{2}\left(d_{0}-1\right)-\frac{1}{2} h_{0} u_{2}\left(d_{0}\right)+u_{2}\left(d_{0}\right)\right) .
$$

Inserting (3.8), (3.9) into (3.4) and then equating the terms do not depend on $h_{0}$ of both sides, we have

$$
u_{2}\left(d_{0}\right)^{2}=u_{2}\left(d_{0}\right) u_{2}\left(d_{0}-1\right)
$$

which implies $u_{2}\left(d_{0}\right) \in \mathbb{C}$. Thus,

$$
E_{0}\left(d_{0}, h_{0}\right)=\alpha\left(\frac{h_{0}}{2}+\beta\right) \quad \text { and } \quad F_{0}\left(d_{0}, h_{0}\right)=-\frac{1}{\alpha}\left(\frac{h_{0}}{2}-\beta\right) \quad \text { for some } \beta \in \mathbb{C} .
$$

These along with

$$
\left[h_{i}, e_{0}\right] \cdot 1=2 e_{i} \cdot 1 \quad \text { and } \quad\left[h_{i}, f_{0}\right] \cdot 1=-2 f_{i} \cdot 1, \quad \forall i \in \mathbb{Z}
$$

give

$$
E_{i}\left(d_{0}, h_{0}\right)=\lambda^{i} \alpha\left(\frac{h_{0}}{2}+\beta\right) \quad \text { and } \quad F_{i}\left(d_{0}, h_{0}\right)=-\frac{\lambda^{i}}{\alpha}\left(\frac{h_{0}}{2}-\beta\right), \quad \forall i \in \mathbb{Z} .
$$

And from $\left[d_{i}, e_{0}\right] \cdot 1=0$ we see that for any $i \in \mathbb{Z}, p_{i}\left(h_{0}\right)=i \gamma$ for some $\gamma \in \mathbb{C}$. Then in this case, $M \cong \Theta(\lambda, \alpha, \beta, \gamma)$. 


\section{REFERENCES}

[1] P. Batra, V. Mazorchuk, Blocks and modules for Whittaker pairs, J. Pure Appl. Algebra 215, 15521568 (2011).

[2] Y. Billig, A category of modules for the full toroidal Lie algebra, International Mathematics Research Notices, 46pp (2006).

[3] Y. Cai, H. Tan, K. Zhao, Module structure on $U(\mathfrak{h})$ for Kac-Moody algebras (in Chinese), Sci. Sin. Math. 47(11), 1491-1514 (2017).

[4] Y. Cai, K. Zhao, Module structure on $U(\mathfrak{h})$ for basic Lie superalgebras, Toyama Math. J. 37, 55-72 (2015).

[5] H. Chen, J. Han, A class of non-weight modules over the Virasoro algebra, arXiv:1712.01436v1.

[6] H. Chen, X. Guo, Non-weight Modules over the Heisenberg-Virasoro and W(2,2) algebras, J. Algebra Appl. 16, 1750097 (2017).

[7] H. Chen, X. Guo, A new family of modules over the Virasoro algebra, J. Algebra 457, 73-105 (2016).

[8] Q. Chen, Y. Cai, Modules over algebras related to the Virasoro algebra, Internat. J. Math. 26, 1550070 (2015).

[9] Q. Chen, Y. Yao, Non-weight modules over algebras related to the Virasoro algebra, J. Geom. Phys. 134, 11-18 (2018).

[10] S. Cheng, N. Lam, Finite conformal modules over the $N=2,3,4$ superconformal algebras, J. Math. Phys. 42(2), 906-933 (2001).

[11] S. Eswara Rao, C. Jiang, Classification of irreducible integrable representations for the full toroidal Lie algebras, J. Pure Appl. Algebra 200, 71-85 (2005).

[12] Y. Gao, N. Hu, D. Liu, Representations of the affine-Virasoro algebra of type $A_{1}$, J. Geom. Phys. 106, 102-107 (2016).

[13] J. Han, Submodule structure of $\mathbb{C}[s, t]$ over $\operatorname{Vir}(0, b)$, arXiv:1708.07272v2.

[14] J. Han, Q. Chen, Y. Su, Modules over the algebras Vir (a,b), Linear Algebra Appl. 515, 11-23 (2017).

[15] C. Jiang, H. You, Irreducible representations for the affine-Virasoro Lie algebras of type $B_{l}$, Chinese Ann. Math. Ser. B 25(3), 359-368 (2004).

[16] V. G. Kac, Highest weight representations of conformal current algebras, Symposium on Topological and Geometric Methods in Field Theory, Espoo, Finland, World Scientific, 3-16 (1986).

[17] G. Kuroki, Fock space representations of an affine Lie algebras and integral representations in the Wess-Zumino-Witten models, Comm. Math. Phys. 142(3), 511-542 (1991).

[18] X. Liu, M. Qian, Bosonic Fock representations of the affine-Virasoro algebra, J. Phys. A 27(5), 131-136 (1994).

[19] R. Lü, X. Guo, K. Zhao, Irreducible modules over the Virasoro algebra, Doc. Math. 16, 709-721 (2011).

[20] R. Lü, K. Zhao, Irreducible Virasoro modules from irreducible Weyl modules, J. Algebra 414, 271-287 (2014). 
[21] V. Mazorchuk, E. Weisner, Simple Virasoro modules induced from codimension one subalgebras of the positive part, Proc. Amer. Math. Soc. 142(11), 3695-3703 (2012).

[22] V. Mazorchuk, K. Zhao, Simple Virasoro modules which are locally finite over a positive part, Selecta Math. (N.S.) 20(3), 839-854 (2014).

[23] J. Nilsson, Simple $\mathfrak{s l}_{\mathfrak{n}+\mathbf{1}^{-}}$-module structures on $U(\mathfrak{h})$, J. Algebra 424, 294-329 (2015).

[24] J. Nilsson, $U(\mathfrak{h})$-free modules and coherent families, J. Pure Appl. Algebra 220, 1475-1488 (2016).

[25] H. Tan, K. Zhao, Irreducible Virasoro modules from tensor products (II), J. Algebra 394, 357-373 (2013).

[26] H. Tan, K. Zhao, $\mathfrak{W}_{\mathfrak{n}}^{+}$and $\mathfrak{W}_{\mathfrak{n}}$-module structures on $U\left(\mathfrak{h}_{\mathfrak{n}}\right)$, J. Algebra 424, 257-375 (2015).

[27] L. Xia, N. Hu, Irreducible representations for Virasoro-toroidal Lie algebras, J. Pure Appl. Algebra 194, 213-237 (2004).

Department of Mathematics, Shanghai Maritime University, Shanghai, 201306, China.

E-mail address: chenqf@shmtu.edu.cn

School of Mathematical Sciences, Tongui University, Shanghai, 200092, China.

E-mail address: jzhan@tongji.edu.cn 